\title{
Hyperspectral Feature Selection and Fusion for Detection of Chicken Skin Tumors
}

\author{
Songyot Nakariyakul and David Casasent \\ Department of Electrical and Computer Engineering \\ Carnegie Mellon University, Pittsburgh, PA 15213
}

\begin{abstract}
We consider a feature selection method to detect skin tumors on chicken carcasses using hyperspectral reflectance data. This allows for faster data collection than does fluorescence data. A chicken skin tumor is an ulcerous lesion region surrounded by a region of thickened-skin. Detection of chicken tumors is a difficult detection problem because the tumors vary in size and shape; some tumors appear on the side of the chicken. In addition, different areas of normal chicken skin have a variety of hyperspectral response variations, some of which are very similar to the spectral responses of tumors. Similarly, different tumors and different parts of a tumor have different spectral responses. Thus, proper classifier training is needed and many false alarms are expected. Since the spectral responses of the lesion and the thickened-skin regions of tumors are considerably different, we train our feature selection algorithm to detect lesion regions and to detect thickened-skin regions separately; we then process the resultant images and we fuse the two HS detection results to reduce false alarms. Our new forward selection and modified branch and bound algorithm is used to select a small number of $\lambda$ spectral features that are useful for discrimination. Initial results show that our method offers promise for a good tumor detection rate and a low false alarm rate.
\end{abstract}

Keywords: chicken tumor detection, feature reduction, feature selection, hyperspectral data, product inspection, tumor detection.

\section{INTRODUCTION}

Hyperspectral (HS) image data is high-dimensional data that contains more than a hundred images in narrowly spaced spectral bands $(\lambda)$. It has been shown that use of hyperspectral information is useful for detection of objects in military applications such as detecting military vehicles [1, 2, 23] and mines [3, 23], for land use applications [4], and for many USDA product inspection applications [5-12]. This occurs since HS data provides spectral information that uniquely characterizes and identifies the chemical, moisture, and physical properties of the constituent parts of an input object, scene region, or an agricultural product. Hyperspectral data has successfully classified: internal-damaged almonds from normal ones [5, 24], aflatoxin-infested corn kernels from good ones [6-8], scab vomitoxin and ergosterol in single wheat kernels [9], and fecal contaminated chicken carcasses from clean ones [10-12].

One of the main problems in the classification of high-dimensional data is that there are often not enough samples in the training data. It is generally accepted that the required number of training samples must be at least ten times the number of features (or in this case input $\lambda$ spectral samples per class) [13] if one wants to be able to accurately predict the class of an unknown sample. This phenomenon is known as the curse of dimensionality. Thus, use of hyperspectral data requires more than a thousand training samples per class in order to cope with the curse of dimensionality. In general, this number of samples is quite difficult to obtain. Thus, it is necessary to reduce the number of features by either feature extraction or feature selection techniques. Feature extraction refers to algorithms that map all of the original features into a few features (each of which is a function of all original features), and feature selection refers to algorithms that select a small subset of the input feature set (use of only several $\lambda$ features) to use for classification. Feature selection is preferable because it provides faster data acquisition and a less expensive system. We consider a new feature selection algorithm developed earlier at Carnegie Mellon University [5]

In this paper, we consider the use of feature selection on hyperspectral images for the detection of skin tumors in HS reflectance images of chicken carcasses. A chicken skin tumor is a round ulcerous lesion region surrounded by a region of thickened-skin [14]. Figs. 1 and 2 show the $486.9 \mathrm{~nm}$ wavelength band images of three chicken carcasses with all tumors numbered and marked by rectangles. The images in HS data are gray-scale; the image in each $\lambda$ spectral band is affected by the skin color, shading, and slope of each local region of the carcass. Figs. 3a to $3 \mathrm{~d}$ are enlarged images of 4 tumors in Fig. 1. The lesion regions of chicken skin tumors in Figs. 1-2 vary in size from $2 \times 4$ pixels to more than 
$25 \times 15$ pixels. In a single gray scale HS image at one $\lambda$, such as Fig. 1 , the central ulcerous lesion region of a tumor appears as a dark-gray region, as seen in Figs. 3a to 3d, and the thickened-skin region immediately surrounding the lesion region appears as bright rings. When tumors occur on the side of the carcass, they appear elliptical and are very small. Such tumors are tumors numbered 4 and 5 in Fig. 2. Thus, detecting chicken skin tumors is a difficult problem.
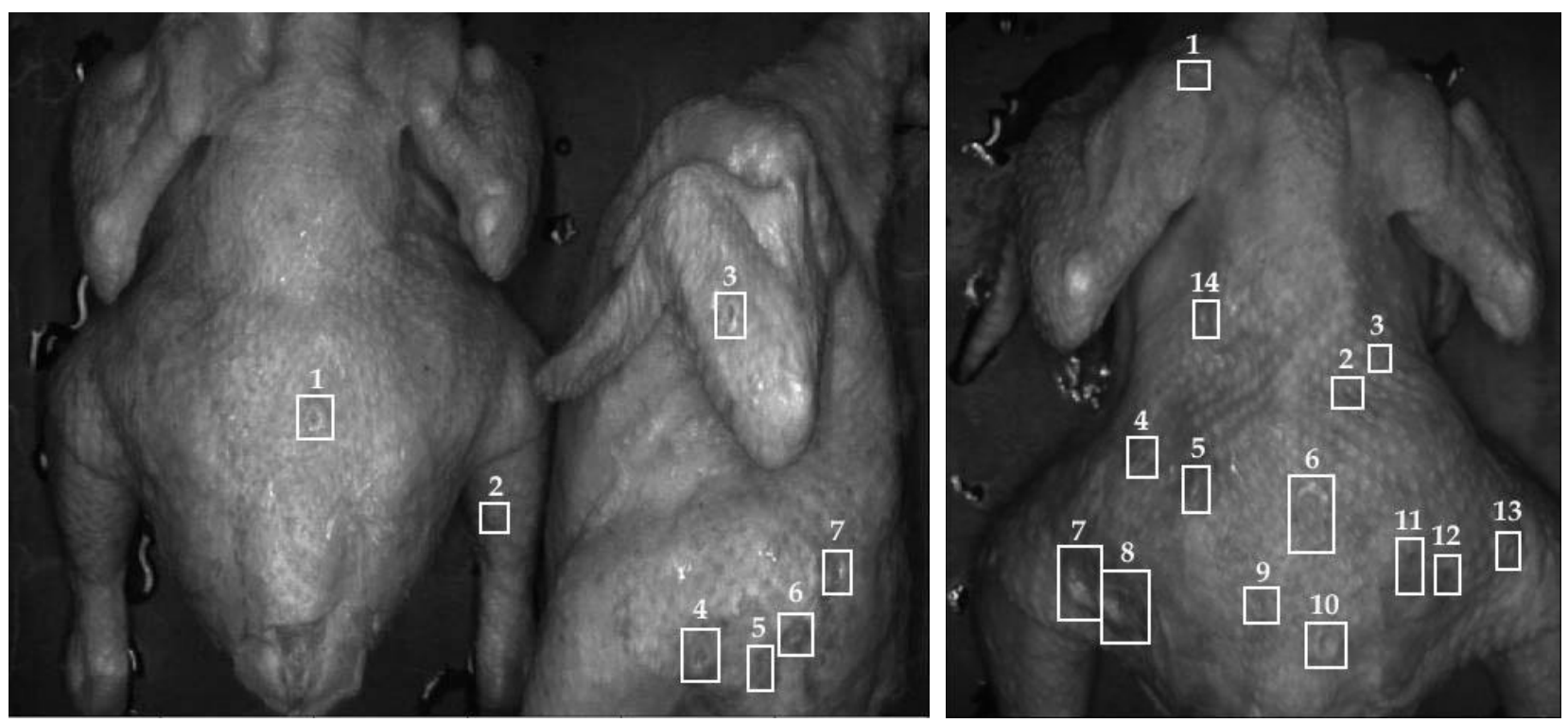

Figure 1: The $486.9 \mathrm{~nm}$ wavelength band image of first HS images with all 7 tumors numbered and marked by rectangles. images with all 14 tumors numbered and marked by rectangles.

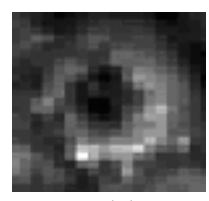

(a)

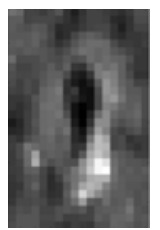

(b)

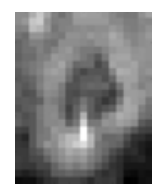

(c)

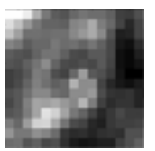

(d)

Figure 3: Enlarged images of the tumors numbered 1, 3, 4 and 6 respectively in Figure 1.

Prior work on detection of chicken skin tumors using HS data considered statistical properties of HS image local regions in three selected bands $(\lambda)$ [15]. Principle component analysis was applied to hyperspectral images of normal and tumor regions on 8 chicken carcasses. The first 10 PCA eigenimages were calculated for each of the 8 chicken HS image sets. The eigenimage with the best contrast and difference between the tumor and normal regions was chosen and the three bands in its eigen decompositions with the largest coefficients were used as the selected bands. The $465 \mathrm{~nm}$, $575 \mathrm{~nm}$, and $705 \mathrm{~nm}$ bands were used. These bands lie in the B, G, and R portions of the spectrum, allowing use of a 3color camera for detection with $\pm 10 \mathrm{~nm}$ filters in each region. A square grid with a local mesh size of $64 \times 64$ pixels was placed over each final combined image with each pixel corresponding to a sample area of $0.1 \mathrm{~mm}^{2}$. Statistical features (mean, skewness, kurtosis, and coefficient of variation, defined as (standard deviation / mean) $\times 100$ ) were calculated for the pixels in each square in this grid and used as inputs to fuzzy classifiers [15]. The training set for the fuzzy classifiers consisted of 100 normal and 52 tumor regions (each was 64×64 pixels). The fuzzy classifiers classify each grid region as normal or tumorous skin. Use of three features (coefficient of variation, skewness, and kurtosis) gave detection rates of $91 \%$ and $86 \%$ for normal and tumorous skin tissue region, respectively (44 of 51 test set tumors were detected). We found the grid size too large, since the lesion regions of 5 of the 21 skin tumors in our database consist of only 8 to 20 pixels. This emphasizes the need to classifying each pixel individually in our data. Kim et al [16] approached the problem differently using HS fluorescence imaging. They computed the maximum intensities, slopes, and ratios of maximum intensities in several specific wavelength bands for each pixel and used them as features for a linear classifier. They used 48 tumor and 65 normal skin pixel samples for training the spectral classifier. Their three $\lambda$ features were chosen by inspection of the training data; as a result, these features are not guaranteed to give the best solution. A 
simple unspecified linear classifier was used to classify image pixels into either tumor or normal class. We note that the data may not be linearly separable. Normal-class pixels that were misclassified by the linear classifier as tumor-class pixels are referred to as false alarms. Spatial image processing (size and ratio of major and minor axis of blob regions) was applied to the resultant binary 2-class image to remove false alarms. 31 of 41 skin tumors (76\%) in 10 HS image sets were detected with 12 false alarm regions. Our 2 images of 3 carcasses were in their set of HS images; but a different HS system (reflectance rather than fluorescence) was used for our data as more light results.

Our database contains HS reflectance images in the first 65 spectral bands $(\lambda)$ out of 112 ranging from $\lambda=447.3$ to $733.5 \mathrm{~nm}$. We show (in Fig. 4) the spectral responses of four of the tumors and four of the normal skin regions of the carcass in Fig. 1. These are the responses at one pixel in the lesion regions of tumors numbered 1, 3, 4 and 6 in Fig. 1 (or Figs. 3a to 3d, respectively) and in various normal skin regions of Fig. 1. From Fig. 4, we see that the spectra of the lesion regions of tumors have similar relative shapes but varying intensities. This is expected because tumors on the side of the carcass (e.g. tumor number 6) reflect the incident light away from the HS sensor, resulting in lower intensity responses than those from tumors in the middle of the carcass (e.g. tumors numbered 1, 3, and 4). This emphasizes the need to normalize the response at every pixel in the database before training or testing. Thus, the response at each data pixel is normalized by dividing its response by its average response over all wavelengths. Fig. 5 shows the normalized version of the spectra in Fig. 4. The spectral responses of the lesion regions of tumors and the normal skin regions are different. The spectral response from other normal skin regions is much closer to the lesion response curves. We found that the spectral responses of the lesion regions of different tumors are not exactly the same. We found that the responses of different normal skin regions vary considerably; thus one must carefully select the training set pixel database to represent normal skin regions.
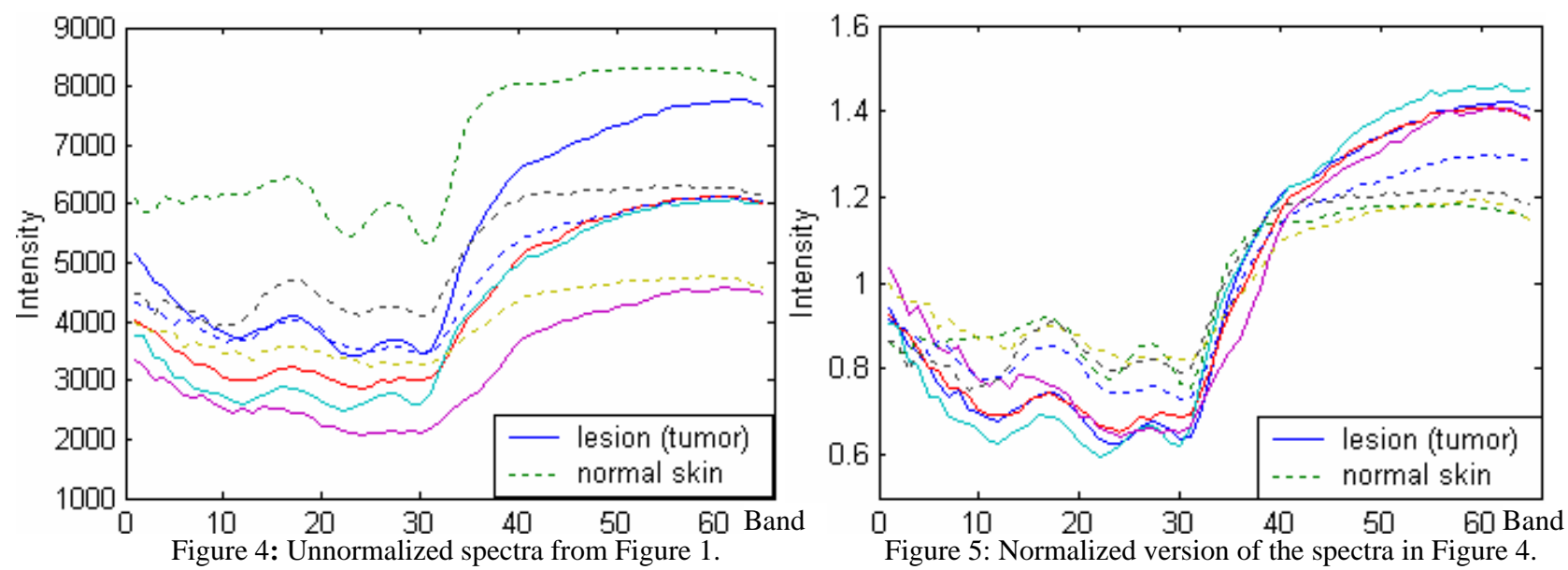

We were unable to detect only the tumor regions using HS data with a single set of $\lambda$ bands without a large number of false alarms. Thus, we use HS processing to separately detect the central lesion regions and the outer thickened-skin regions of the tumors. A binary image was produced for each case. These images were then fused and morphologically processed to produce our final images. Fusion was found to be necessary to reduce false alarms. The use of fusion is new and has not previously been employed in HS data processing. Fig. 6 shows the normalized spectra of some pixels in the lesion and thickened-skin regions of tumor 1 in Fig. 1. It is clear from Fig. 6 that the lesion and thickened-skin regions of tumors have different HS responses. Thus, we use different sets of feature bands to detect each. To do this, we select a portion of the lesion region and normal skin region pixels from the chicken images as the pixel training/test set or the lesion pixel database. We also create a thickened-skin pixel database that includes a portion of the thickenedskin region and the normal skin region pixels from the chicken images; this is the pixel training/test set for thickenedskin. We train our feature selection algorithm on the lesion pixel database and on the thickened-skin pixel database; the results are used to detect the lesions and the thickened-skin regions of tumors, respectively.

We must first select the optimal features $(\lambda s)$ to use. The only optimal feature selection algorithms are exhaustive search and branch and bound (BB) [17]. An exhaustive search finds the best subset of $m$ features out of $n$ by evaluating a criterion function $\mathrm{J}$ for all possible feature combinations and selects the best set. In many hyperspectral image cases that have more than a hundred features $(\lambda s)$, an exhaustive search is very time consuming and prohibitive. The BB algorithm is more efficient because it avoids an exhaustive search of the whole search space by rejecting many subsets 
that are guaranteed to be sub-optimal, and it guarantees that the selected subset is the globally optimal solution for any criterion function that satisfies monotonicity. A modified branch and bound (MBB) algorithm developed by Casasent and Chen [5] modifies the feature ordering and the BB algorithm and provides a more efficient way to search the subsets. Thus, it is faster than BB and much faster than an exhaustive search. However, for general HS data with more than a hundred feature bands $(\lambda)$, the computational load for the BB and MBB algorithms is also impractical for feature selection. This emphasizes the need to reduce the dimensionality of the problem before we apply the BB or MBB algorithms. We use the forward selection (FS) algorithm [18, p. 490] to select 30 initial features and then use the MBB algorithm to select a number of final features (three or four features at most) to use. This is our FS/BB algorithm [5]. Different features $(\lambda s)$ were used to detect lesion and thickened-skin regions of the tumors. We use a KNN classifier to obtain classification rate $\left(\mathrm{P}_{\mathrm{C}}\right)$ pixel data and to obtain pixel images for lesion and thickened-skin processing.

Sect. 2 describes the database used. Sect. 3 discusses the feature selection algorithms used. Methods and test results are presented in Sects. 4 and 5.

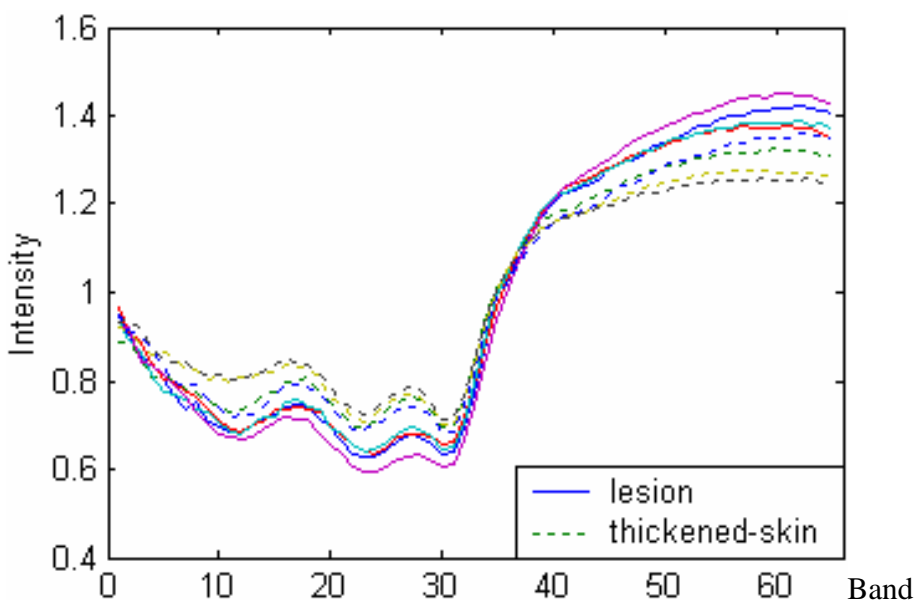

Figure 6: Normalized spectra of pixels in the lesion and thickened-skin regions of tumor 1 in Figure 1.

\section{DATABASE}

Chicken carcasses with skin tumors were sent for HS processing to the ARS Instrumentation and Sensing Laboratory (ISL) in Maryland. The hyperspectral (HS) imaging system used consisted of a CCD camera, a spectrograph, a sample transport mechanism, and lighting sources [15]. More details on the ISL hyperspectral imaging system are provided elsewhere [19]. The locations of tumors were verified by a Food Safety and Inspection Service (FSIS) veterinarian. Two HS cubes were provided to us for initial testing (a HS cube contains a series of images in narrowly spaced spectral bands $(\lambda)$, where each image corresponds to the image obtained at one specific frequency band.) Each HS cube consists of 65 spectral band images ranging from $\lambda=447.3$ to $733.5 \mathrm{~nm}$. The first HS cube contains two chicken carcasses with a total of 7 tumors on them. The size of each image is $460 \times 600$ pixels; the $465 \mathrm{~nm}$ wavelength band image from this HS cube was shown in Fig. 1. The second HS cube has a chicken carcass with 14 skin tumors. The size of each image is $460 \times 400$ pixels; the $486.9 \mathrm{~nm}$ wavelength band images were shown in Figs. 1 and 2 . Tumor number 14 in Fig. 2 was identified by the FSIS veterinarian as normal tissue, but Kim et al [17] stated in their paper that it was a tumor. We agree (so does our feature selection algorithm). Several tumors in the second HS cube in Fig. 2 are small (the lesion regions of tumors 4 and 5 contain only 8 pixels), compared to those in the first HS cube (Fig. 1). Thus, we do not expect our feature selection algorithm to detect them.

In general, one could select the pixel training and test set pixel database from one HS cube and train the feature selection algorithm on them. One could then apply the feature selection results to the second HS cube, that has not been trained on before. However, our database is limited; we have only three chicken carcasses available for training and testing. We thus selected a portion of the skin tumors from only the first HS cube and the normal skin regions from both HS cubes for pixel training and testing. This was necessary to reduce false alarms in the second image, since some normal skin regions in the second HS cube have very different spectral responses from those in the first HS cube. Therefore, it is necessary to select normal skin region training data from both HS cubes. The tumor regions in both images seem to have similar spectral responses. We want to have a number of tumors (with no training set pixels in them used) present. For the lesion pixel database, we extracted the $65 \lambda$ band spectral responses for 50 pixels from 4 of 
the 7 ulcerous lesion regions of the tumors numbered 1, 3, 4, and 6 in Fig. 1, since the regions of these tumors are more well-defined. We labeled them as "tumor" class, and used them for the pixel training set. The $65 \lambda$ band spectral responses for 50 pixels from the lesion regions of 3 different tumors (2, 5, and 7 in Fig. 1) were used for the pixel test set of target (tumor) pixel data. With 50 tumor pixels for the pixel training set and 65 spectral features, this represents high-dimensional data. We extracted the $65 \lambda$ band spectral responses for 360 pixels from normal skin regions of 2 of the 3 carcasses and labeled them as "normal" class (no training samples were taken from the left carcass in HS image 1). Since different areas of normal chicken skin have different spectral responses, we used more normal-class samples than tumor-class samples. We used half (180 samples) of these normal samples as the pixel training set and half (180 samples) in the pixel test set for normal class pixel data. 300 of the 360 normal samples were selected from various normal skin regions of the carcass in HS cube 2 that have very different spectral responses. These regions include pale skin, pinkish skin, skin covering bony joints, and the shadow area under the wings. 60 of the 360 normal skin samples were chosen from the normal skin regions of the right carcass in the first HS cube, since this carcass displays the side of the chicken. Thus, some regions in this carcass are not present in other carcasses, and they have different spectral responses.

For the thickened-skin pixel database, we extracted the $65 \lambda$ band spectral responses for 50 pixels from the thickened-skin regions surrounding the same four tumors chosen for the pixel training set for the lesion pixel database, labeled them as tumor class, and used them for the thickened-skin pixel training set. We extracted $65 \lambda$ band spectral responses for 50 pixels from the thickened-skin regions surrounding the same three tumors chosen for the pixel test set for our lesion pixel database, and used them for our thickened-skin pixel test set. The normal skin pixel training and test set pixel databases were the same for the lesion and thickened-skin case.

\subsection{Forward selection (FS) algorithm}

\section{FEATURE SELECTION ALGORITHMS}

We assume that a maximum of 3 spectral $(\lambda)$ bands will be used. The FS method first selects the best single feature and then adds one feature at a time, which in combination with the first selected feature maximizes some criterion function J, etc. We use the Bhattacharya distance as the criterion function, i.e.,

$$
\mathrm{J}=\frac{1}{8}\left(\boldsymbol{\mu}_{1}-\boldsymbol{\mu}_{2}\right)^{\mathbf{T}}\left(\frac{\mathbf{C}_{1}+\mathbf{C}_{2}}{2}\right)^{-1}\left(\boldsymbol{\mu}_{1}-\boldsymbol{\mu}_{2}\right)+\frac{1}{2} \ln \left|\frac{\mathbf{C}_{1}+\mathbf{C}_{2}}{2}\right| / \sqrt{\left|\mathbf{C}_{1}\right|\left|\mathbf{C}_{2}\right|},
$$

where $\mu_{1}$ and $\mu_{2}$ are the mean vectors for the tumor-class and normal-class training samples, and $\mathbf{C}_{1}$ and $\mathbf{C}_{2}$ are the covariance matrices for the tumor-class and normal-class training samples, respectively [20, p. 48]. The Bhattacharya distance is large if the mean difference between two classes is large (the first term in J) and if the variances of the two classes are different (the second term in $\mathrm{J}$ ). To select the best subset of $\mathrm{m}$ features out of $\mathrm{n}$ original features, the number of subsets searched by the FS algorithm is $[(2 n-m+1) m] / 2$, which is much smaller than the number of subsets evaluated in an exhaustive search $(\mathrm{n} ! /[\mathrm{m} !(\mathrm{n}-\mathrm{m}) !])$ or in the branch and bound method. For example, to select the best subset of 3 features out of 65 , the FS algorithm requires searching $[(2 \times 65-3+1) \times 3] / 2=192$ subsets, whereas an exhaustive search requires searching 65!/[3!(65-3)!] = 131,040 subsets. However, the FS method does not examine all possible subsets, so the resulting subset is not guaranteed to produce the optimal set of features nor the best classification rate $\mathrm{P}_{\mathrm{C}}$. The FS method also has the nesting problem, i.e. the subset of four best features chosen by FS contains the subset of three best features chosen by FS, etc. In practice, the best four features may not contain any of the best three features, etc. Recall that the FS algorithm produces a set of ordered features. We thus use the FS algorithm to select 30 initial features (more than the final number of 3 features that we desire to use); and we use the MBB algorithm to select the optimal subset of final features ( 3 features at most) out of these $30 \mathrm{FS}$ features to use in our classifier. This is our FS/MBB algorithm [5].

\subsection{Modified branch and bound (MBB) algorithm}

Since the modified branch and bound (MBB) algorithm developed at CMU [5] uses modifications to the basic branch and bound (BB) algorithm, we thus give a brief description of the basic BB algorithm. To select the best set of $\mathrm{m}$ features out of $\mathrm{n}$ original features, the BB algorithm selects the $\mathrm{n}-\mathrm{m}$ features to be discarded. It creates a search tree with $n-m$ levels with one feature being omitted at each level of the tree. The problem is to select the best path through the tree that yields the largest $\mathrm{J}$. The $\mathrm{BB}$ algorithm assumes monotonicity of $\mathrm{J}$, i.e. $\mathrm{J}$ decreases as we move down the tree; this is logical because more features are omitted as we move down the tree. We use the Bhattacharya distance in (1) as the criterion function. The BB algorithm starts the search at the top of the tree, and all nodes at level-1 are analyzed. For a given level-1 node, it has several nodes below it. The successor node below the level-1 node with the largest $\mathrm{J}$ is analyzed further. The search continues until it reaches the bottom of the tree, the $\mathrm{n}$ - $\mathrm{m}$ level, resulting in one full path through the tree with an initial estimate (a bound B) for the criteria function J. J is then evaluated at other 
level-1 nodes and the process is continued to lower levels of the tree; if $\mathrm{J}<\mathrm{B}$ for a given node, then $\mathrm{J}$ does not have to be evaluated at successor nodes under that node, because $\mathrm{J}$ decreases as we proceed down the tree. This causes the BB algorithm to be fast, as large portions of the tree need not be searched. If $\mathrm{J}>\mathrm{B}$ for a given node (larger $\mathrm{J}$ values are better), paths from that node to the bottom of the tree are explored (as long as their $\mathrm{J}$ remains larger than $\mathrm{B}$ ). When a mother node has a low $\mathrm{J}<\mathrm{B}$, its successor nodes need not be analyzed. Omitting evaluation of $\mathrm{J}$ for a set of successor nodes (when $\mathrm{J}<\mathrm{B}$ at some mother nodes) speeds up the search, and thus BB is more efficient than an exhaustive search. If a new different full path with a $\mathrm{J}>\mathrm{B}$ is found, the bound $\mathrm{B}$ is updated with the new larger value.

A new BB algorithm improvement in the MBB algorithm is to obtain a good initial estimate of B [5]. If we can obtain a good, high initial $\mathrm{B}$, many more subsequent $\mathrm{J}$ values higher up in the tree are less likely to give a $\mathrm{J}>\mathrm{B}$. Therefore, calculations of $\mathrm{J}$ for many paths can hopefully be omitted. The MBB also uses FS or other sub-optimal algorithms to order all $\mathrm{n}$ features from best to worst, and the tree is then constructed with this ordered featured set. An initial estimate of B is calculated using the $\mathrm{m}$ best features ordered by FS. This B bound estimate is higher than one estimated by using non-ordered features. We thus expect a low $\mathrm{J}$ to be obtained at many mother nodes where the better FS features are omitted. When this occurs, a search of all subsets of features below these nodes can then be omitted, and this speeds up the search. Another MBB modification is to use jump starting search levels. The motivation for this is that at the upper levels of the search tree, we do not expect $\mathrm{J}<\mathrm{B}$, since only one or two features are omitted. In MBB, we thus start the BB search ( $J$ evaluation) at level $(n-m) / 4$, because we only expect $J$ to be less than a good initial B estimate when some reasonable number of features are omitted. $\mathrm{J}$ is evaluated for all nodes at this level. If all nodes give $\mathrm{J}>\mathrm{B}$, we jump to level $(\mathrm{n}-\mathrm{m}) / 2$, calculate $\mathrm{J}$ for all of its nodes, and apply the BB search to nodes below all nodes with $\mathrm{J}>\mathrm{B}$. If any node at some level such as $(\mathrm{n}-\mathrm{m}) / 4$ has $\mathrm{J}<\mathrm{B}$, we apply the BB algorithm to all nodes below nodes at that level with $\mathrm{J}>\mathrm{B}$. If all nodes at level $(\mathrm{n}-\mathrm{m})$ have $\mathrm{J}>\mathrm{B}$, then we know that we would have had to evaluate $\mathrm{J}$ at all nodes above that level. This "jump search" algorithm thus saves searching $\mathrm{J}$ at all nodes above that level [5].

\section{METHODOLOGY}

First, the background around the carcass must be removed. We do this by calculating a mask whose value is one (white) on the carcass and zero (black) on the background. To obtain this, we first obtain the unnormalized spectra of the background and several skin regions on the carcass. Unlike the spectral responses of tumors and normal skins, the spectral response of the background does not noticeably vary over all 65 spectral bands. From these training data, we chose to form the difference in responses at each pixel in two bands (bands 30 and 60) that have large differences in their carcass responses. We calculated this difference for each image pixel, formed a pixel difference image, and set the pixels with an unnormalized intensity difference less than 750 to zero and other pixels to one. A similar method was also used for background removal in $[15,16]$. We removed some residual background blob regions by retaining only connected regions with more than 500 pixels (chicken carcasses have more than 500 pixels). We then perform morphological processing to fill in small holes (less than 15 connected pixels) to produce the final mask.

Second, we select the spectral bands to use to locate the lesion and thickened-skin regions of tumors and separate them from normal skin regions. We train our feature selection algorithm on the lesion pixel database and the thickenedskin pixel database; these are used to detect the lesion and thickened-skin regions of tumors, respectively. We use forward selection (FS) to select the 30 best $\lambda$ features out of the 65 available ones, and we then apply the modified branch and bound algorithm to select a number of final features (FS/MBB algorithm). For this database (with only $65 \lambda$ features), it is possible to apply the MBB algorithm directly to the original databases without first reducing the number of features by FS. In general, we do not expect this to be the case. We show in Sect. 5.1 that the two methods (MBB and FS/MBB) give the same set of final features for our pixel databases. To select the best four features out of all 65 features, MBB took more than two hours, while the FS/MBB algorithm (MBB applied to 30 FS features) took less than two minutes on a Pentium IV 1.8GHz computer. Thus, the proposed FS/MBB algorithm is preferable for many HS applications that need to select at least 3 or 4 best features. The MBB algorithm solution is optimal, whereas the FS/MBB algorithm solution is sub-optimal because FS/MBB only gives the best set of 30 features (by FS) and these are not necessarily the optimal set of 30 features. We hope they contain the 3 best features overall. In such cases, we find small differences in the different spectra chosen. This FS/MBB result is expected, since feature selection is an N-P complete problem [21], and only a search over the entire database can give the best solution.

We now address the image processing applied to the binary image produced after selecting the features from the FS/MBB algorithm and applying them (for each image pixel) to a K-nearest neighbor (KNN) classifier. A binary image results, with each pixel classified as one for the tumor class and zero for the normal skin class. We refer to this as the binary pixel classification image. We expect false alarms because normal chicken skin has a variety of hyperspectral responses, some of which are very similar to the spectral responses of tumors at the few $\lambda \mathrm{s}$ used. In addition, we expect 
to have a higher false alarm rate in the binary pixel classification image than in our final classification image result (after morphological processing). This occurs, since the normal skin training samples in the pixel databases do not represent all of the normal skin regions in a full image. Nevertheless, these normal skin false alarms should not occur at a number of adjacent pixels, if we train our system properly. Since a tumor is not a single pixel but is a region that we assume to consist of at least 13 pixels for the lesion region and at least 5 pixels of thickened-skin region, we thus analyze the blob colored [22] version of the binary pixel classification image and omit any pixel blobs that form connected regions with twelve or less pixels for the lesion regions and four or less pixels for the thickened-skin regions. We thus do not expect our feature selection algorithm to detect tumors with small lesion regions (less than 13 pixels) such as tumors 4 and 5 in Fig. 2. This is necessary to reduce false alarms. Furthermore, we use the fact that we do not expect to detect tumors on the edge of the chicken images (we refer to these rules as post processing). We remove such potential false alarm blobs that appear within 10 pixels of the edge of the chicken in the pixel classification images. The two post-processed binary images for the lesion and thickened-skin cases are then fused; the fusion rule is that if any pixel in the lesion pixel image is within 2 pixels of a pixel in the thickened-skin pixel image, we keep and merge the blobs associated with those pixels and we assume those regions are skin tumors. We allow a separation of 2 pixels, since the thickened-skin region does not overlap the lesion region for all tumors and since all lesion and thickened-skin pixels are not expected to be detected. These blobs are the final fused classification image result. Results are shown in Sect. 5.2.

\subsection{Feature selection pixel database results \\ 5.1.1 The lesion pixel database results}

\section{RESULTS AND DISCUSSION}

We used the FS algorithm to reduce the number of original pixel database features from 65 to 30 and then used the MBB algorithm to select the best subsets of one to three features from these $30 \mathrm{FS}$ features (this is our FS/MBB algorithm). We must also decide what $\mathrm{K}$ to use in our KNN classifier. To select $\mathrm{K}$, we keep only one final FS/MBB feature and increase $\mathrm{K}$ to find the best $\mathrm{K}$ to use in the KNN classifier. To quantify performance, the scores for $\mathrm{P}_{\mathrm{C}}$ (percentage of tumor and normal skin pixels correctly classified), $\mathrm{P}_{\mathrm{D}}$ (percentage of tumor pixels detected), and $\mathrm{P}_{\mathrm{FA}}$ (percentage of normal skin pixels misclassified as tumor pixels) are given. The lesion pixel database results are shown in Table 1. From the training set data in Table 1 , we chose $\mathrm{K}=3$ for the KNN classifier for the lesion pixel database, since it gave the $\mathrm{P}_{\mathrm{C}}$ (train) score at the lowest $\mathrm{K}$ (this gives the lowest online calculations). Our objective was to keep $\mathrm{K}$ as small as possible to allow for faster implementation. Table 2 shows the features selected by the FS algorithm (from all 65 original features), the MBB algorithm (applied to all 65 original features), and our FS/MBB algorithm (applied to the 30 FS features) for the lesion pixel database. As seen, the MBB and FS/MBB algorithms select the same features for discrimination. An exhaustive search also gives the same set of features. Thus, our FS/MBB algorithm selects the optimal set of $\lambda$ features for this pixel database. We note that the features selected by the FS algorithm are very different. We also note that none of the three best features ordered by the FS algorithm in Table 2 are present in the best subset of three features selected by the FS/MBB algorithm. This is the nesting problem that the FS algorithm has. The best subset of three features (features 11, 18, and 29) does not contain any feature in the best subset of two features (features 24 and 31) or one feature (feature 17) chosen by the optimal MBB or FS/MBB algorithm. The FS algorithm cannot handle such cases. Thus, an optimal feature selection algorithm (such as the MBB or the FS/MBB algorithm) is needed and the initial $\lambda$ feature reduction algorithm (such as the FS algorithm) should provide a number of starting features that is much larger than the number of final features considered. Thus, one cannot select the best features using only the FS algorithm.

Table 1. $\mathrm{P}_{\mathrm{C}}$ results for different Ks for the KNN classifier using one FS/MBB feature for the lesion pixel database

\begin{tabular}{|l|ccccccc|}
\hline $\mathrm{K}$ & 1 & 3 & 5 & 7 & 9 & 11 & 13 \\
\hline $\mathrm{P}_{\mathrm{C}}$ (train)\% & 87 & 88 & 88 & 88 & 86 & 88 & 88 \\
$\mathrm{P}_{\mathrm{C}}$ (test)\% & 85 & 85 & 85 & 86 & 85 & 83 & 83 \\
\hline
\end{tabular}

Table 2. Best features chosen by three feature selection algorithms for the lesion pixel database.

\begin{tabular}{|c|ccc|}
\hline The number of features & FS & MBB & FS/MBB \\
\hline 1 & 17 & 17 & 17 \\
2 & 17,65 & 24,31 & 24,31 \\
3 & $17,36,65$ & $11,18,29$ & $11,18,29$ \\
\hline
\end{tabular}


After using the FS/MBB algorithm to reduce the number of features from 65 to a low-dimensional space of 1 to 3 features, each sample in the training and test pixel database is fed to the KNN classifier (using the training set pixels as the $\mathrm{KNN}$ database), and the classification rates $\mathrm{P}_{\mathrm{C}}$ for the training and test pixel sets are obtained. In obtaining training set $\mathrm{P}_{\mathrm{C}}$, the training set sample being classified is of course removed from the NNB classifier. Table 3 shows the scores for $\mathrm{P}_{\mathrm{C}}$ (percentage of lesion and normal skin pixels correctly classified), $\mathrm{P}_{\mathrm{D}}$ (percentage of lesion pixels detected), and $\mathrm{P}_{\mathrm{FA}}$ (percentage of normal skin pixels misclassified as tumor pixels) for the pixel test data using the features chosen by the FS and the FS/MBB algorithms as the number of final features is increased. When 2 or 3 features are used, the test set $\mathrm{P}_{\mathrm{C}}$ scores $(90 \%$ and $94 \%)$ using our $\mathrm{FS} / \mathrm{MBB}$ algorithm are noticeably higher than those $\left(\mathrm{P}_{\mathrm{C}}=84 \%\right.$ and $\left.90 \%\right)$ using the features selected by the FS algorithm. Thus, our optimal FS/MBB feature selection algorithm is needed and does provide better $P_{C}$ etc results. We keep three final FS/MBB features for the lesion pixel database, 4 features may produce higher $\mathrm{P}_{\mathrm{C}}$ scores. From Table 3, we note that low $\mathrm{P}_{\mathrm{FA}}$ scores of 2-3\% are obtained. Image results are presented and discussed in Sect. 5.2.

Table 3. Pixel test data results for features chosen using the FS and FS/MBB algorithms for the lesion pixel database

\begin{tabular}{|c|ccc|ccc|}
\hline \# features & \multicolumn{3}{|c|}{ FS algorithm } & \multicolumn{3}{c|}{ FS/MBB algorithm } \\
& $\mathrm{P}_{\mathrm{C}} \%$ & $\mathrm{P}_{\mathrm{D}} \%$ & $\mathrm{P}_{\mathrm{FA}} \%$ & $\mathrm{P}_{\mathrm{C}} \%$ & $\mathrm{P}_{\mathrm{D}} \%$ & $\mathrm{P}_{\mathrm{FA}} \%$ \\
\hline 1 & 84 & 42 & 4 & 84 & 42 & 4 \\
2 & 84 & 40 & 4 & 90 & 62 & 3 \\
3 & 90 & 62 & 2 & 94 & 84 & 3 \\
\hline
\end{tabular}

\subsubsection{The thickened-skin pixel database results}

Table 4 compares the $\mathrm{P}_{\mathrm{C}}$ scores (percentage of thickened-skin and normal skin pixels correctly classified) using the best feature selected by the FS/MBB algorithm as $\mathrm{K}$ in the KNN classifier is increased for the thickened-skin pixel database. In obtaining training set $\mathrm{P}_{\mathrm{C}}$, the training set sample under test is removed from the NNB classifier. From Table 4, the $P_{C}$ score for the training set pixels is highest when $K$ is 9,11 , or 13 . We use the lowest $K$ (K=9) choice to reduce online calculations. For the thickened-skin pixel database, Table 5 compares the features selected by: the FS algorithm (from all 65 original features), the MBB algorithm (applied to all 65 original features), and our FS/MBB algorithm (from the best 30 FS features). As we can see from Table 5, the same best single feature (feature 34) is chosen by all algorithms; but for future choices, the features chosen differ, but not as much as in Table 2. For all choices, the MBB and FS/MBB algorithms yield the same features and (for this database) our algorithm is again optimal. When three features are used, the FS algorithm chose only one (feature 11) of the optimal features chosen by the FS/MBB algorithm, but the other two features are close in $\lambda$ (feature 18 vs 16 and feature 33 vs 34). We also see the nesting problem in the FS algorithm; the best subset of three features (features 11, 16, and 33) chosen by the MBB or FS/MBB algorithm does not contain any feature in the best subset of two features (features 18 and 61). The FS algorithm cannot make such choices. Table 6 compares the scores for $\mathrm{P}_{\mathrm{C}}, \mathrm{P}_{\mathrm{D}}$, and $\mathrm{P}_{\mathrm{FA}}$ for the pixel test data using the features chosen by the FS and the FS/MBB algorithms as the number of final features selected is increased. $\mathrm{P}_{\mathrm{C}}$ (test) score are all better (or comparable) for our FS/MBB algorithm. We note that all scores increases as the number of features $(\lambda)$ used increases. We expect that the use of four or more FS/MBB features will increase $P_{C}$ further. However, we set a maximum of three wavelength bands for real-time implementation.

Table 4. $\mathrm{P}_{\mathrm{C}}$ results for different Ks for the KNN classifier using one FS/MBB feature for the thickened-skin pixel database

\begin{tabular}{|l|ccccccc|}
\hline $\mathrm{K}$ & 1 & 3 & 5 & 7 & 9 & 11 & 13 \\
\hline $\mathrm{P}_{\mathrm{C}}$ (train)\% & 70 & 74 & 75 & 79 & 80 & 80 & 80 \\
$\mathrm{P}_{\mathrm{C}}$ (test)\% & 74 & 76 & 78 & 79 & 78 & 79 & 80 \\
\hline
\end{tabular}

Table 5. Best features chosen by three feature selection algorithms for the thickened-skin pixel database.

\begin{tabular}{|c|ccc|}
\hline The number of features & FS & MBB & FS/MBB \\
\hline 1 & 34 & 34 & 34 \\
2 & 18,34 & 18,61 & 18,61 \\
3 & $11,18,34$ & $11,16,33$ & $11,16,33$ \\
\hline
\end{tabular}


Table 6. Pixel test data results for features chosen using the FS and FS/MBB algorithms for the thickened-skin pixel database

\begin{tabular}{|c|ccc|ccc|}
\hline \# features & \multicolumn{3}{|c|}{ FS algorithm } & \multicolumn{3}{c|}{ FS/MBB algorithm } \\
& $\mathrm{P}_{\mathrm{C}} \%$ & $\mathrm{P}_{\mathrm{D}} \%$ & $\mathrm{P}_{\mathrm{FA}} \%$ & $\mathrm{P}_{\mathrm{C}} \%$ & $\mathrm{P}_{\mathrm{D}} \%$ & $\mathrm{P}_{\mathrm{FA}} \%$ \\
\hline 1 & 82 & 30 & 4 & 82 & 30 & 4 \\
2 & 85 & 64 & 9 & 86 & 68 & 9 \\
3 & 87 & 68 & 8 & 90 & 70 & 4 \\
\hline
\end{tabular}

\subsection{Image detection results}

\subsubsection{The first HS image cube}

The three chosen features (features 11, 18 and 29) from Table 2 for the lesion pixel database and the KNN classifier $(\mathrm{K}=3)$ were applied to all pixels in the first HS images. Fig. 7a shows the binary classification image using the lesion features for HS image one. We blob color this image, analyze the pixel size of each blob, omit blobs $<13$ pixels in size and blobs that lie within 10 pixels of the edge of the chicken (there are 30 such blobs in Fig. 7a), since we do not expect to detect tumors on the edge of chicken images. The resultant image is shown in Fig. $7 \mathrm{~b}$ (detected tumors are noted by rectangles). The number of false alarms in Fig. 7a is significantly reduced in Fig. 7b. In Fig. 7b, all 7 of the tumors are detected, but 15 false alarm regions are still present. Thus, use of the thickened-skin pixel features is necessary to reduce false alarms. Using only the lesion pixel database or the thickened-skin pixel database for training and testing is not recommended.

The three chosen features (features 11, 16, and 33) from Table 5 for the thickened-skin pixel database were used in the KNN classifier $(\mathrm{K}=9)$ and applied to the first HS images. Thickened-skin regions of each tumor vary in size from 5 to 100 pixels. Thus, we omit any white pixels in the binary classification image which are part of a connected region of four or less pixels. The same binary processing was used. Figs. $8 \mathrm{a}$ and $8 \mathrm{~b}$ show the binary classification results before and after binary image processing using the thickened-skin pixel features. The number of false alarm regions in Fig. 8a is greatly reduced to 95 in Fig. 8b. In Fig. 8b, all 7 tumors are again detected and marked by rectangles. There are more false alarms in the binary pixel classification output image for the thickened-skin features (Fig. 8a) than for the lesion features (Fig. 7a), as we expected from its higher $\mathrm{P}_{\mathrm{FA}}$ score on the pixel database. We then fuse the post-processed binary classification image results for the two feature cases (Figs. 7b and 8b) to reduce FAs. We retain pixel blobs in the binary lesion image that are within 2 pixels of pixel blobs in the thickened-skin binary image and call this a skin tumor. Using this rule, we obtain the final classification image result in Fig. 9. In the final classification image in Fig. 9, we have detected all 7 tumors marked by rectangles and have only two false alarms marked by circles. From an analysis of the color version of this chicken image, we believe that the false alarm at the bottom right in Fig. 9 is in fact a tumor, but the FSIS veterinarian failed to verify it. Fusion of the binary pixel classification images significantly reduces the number of false alarms from more than 15 in Fig. 7b and more than 95 in Fig. $8 b$ to only 2 in Fig. 9.

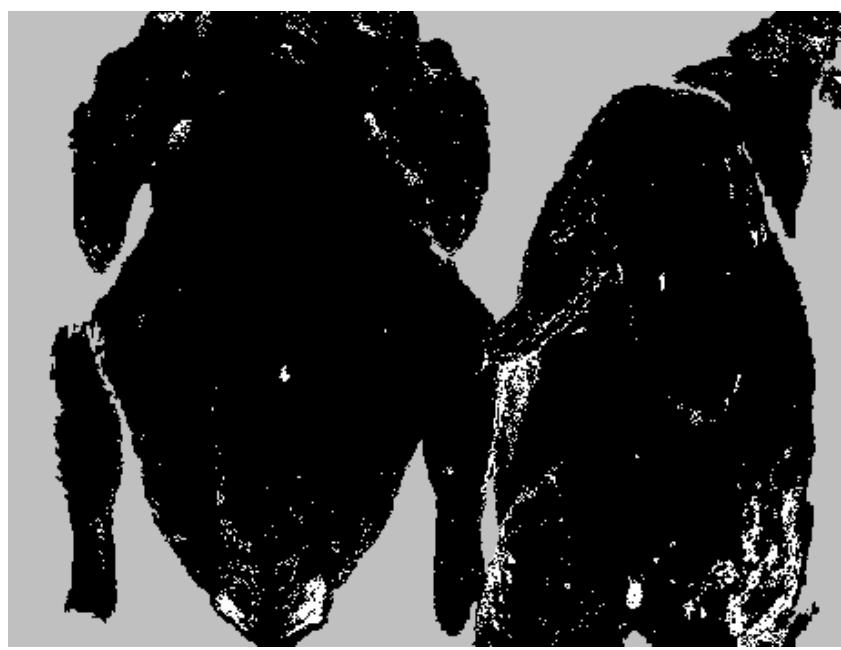

(a) Before binary processing

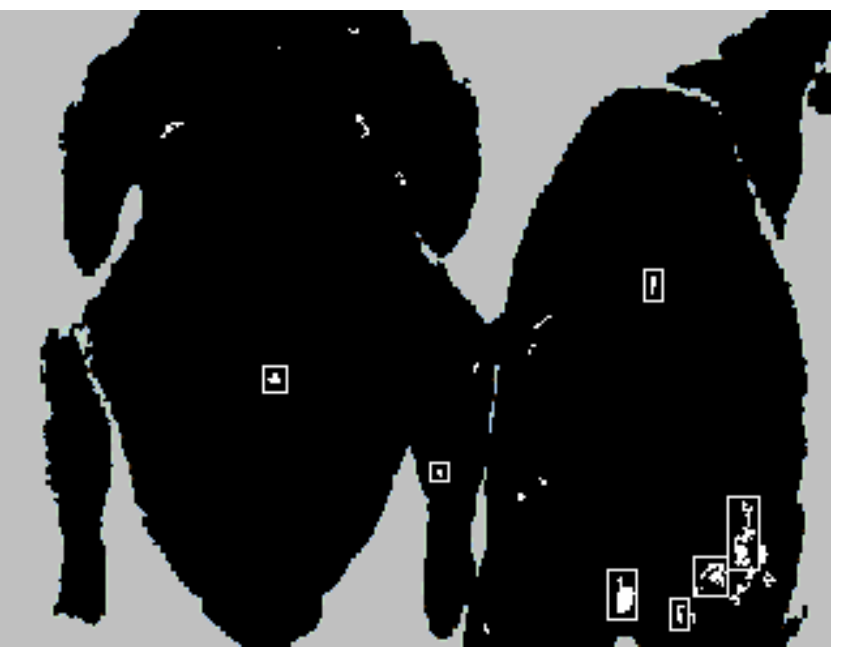

(b) After binary processing

Figure 7: Detection results using the lesion features on the first HS image with detected tumors marked by rectangles. 


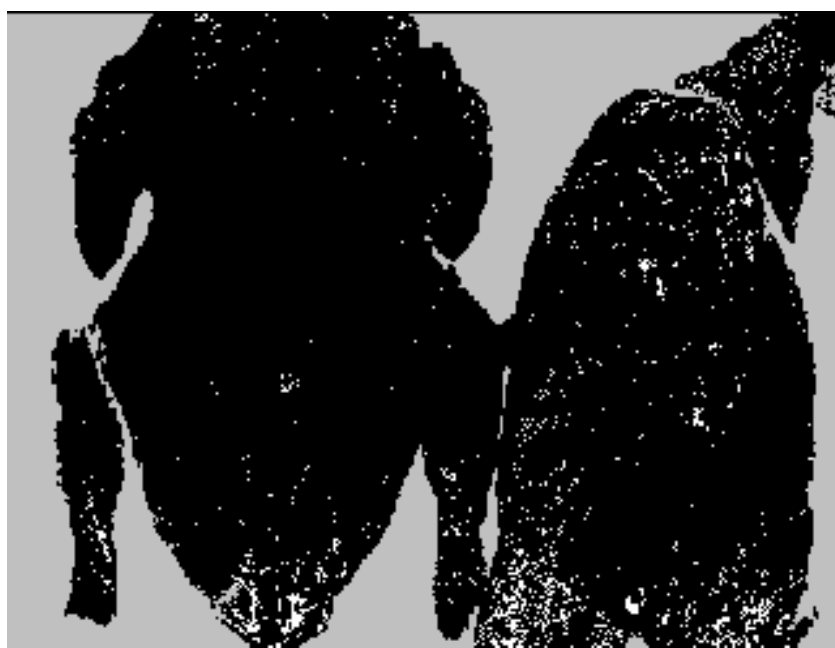

(a) Before binary processing

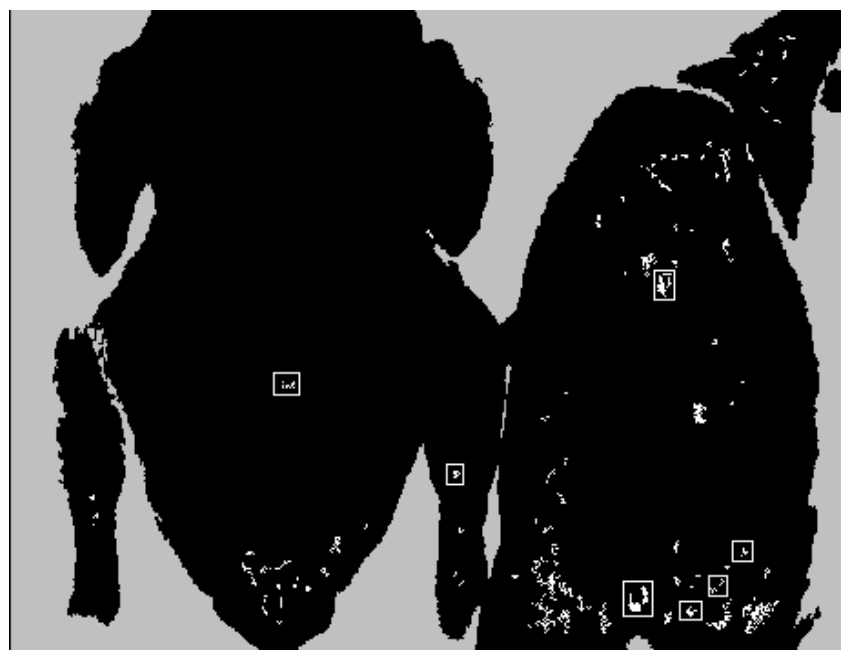

(b) After binary processing

Figure 8: Detection results using the thickened-skin features on the first image with detected tumors marked by rectangles.

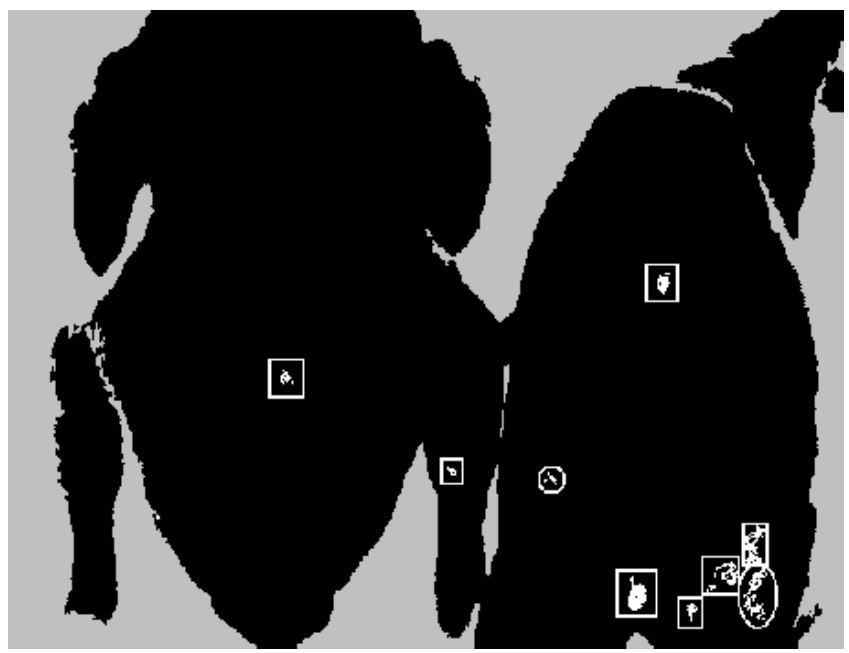

Figure 9: Final fused classification image results for the first HS image with detected tumors marked by rectangles and false alarms marked by circles.

\subsubsection{The second HS image cube}

The chosen features for the lesion pixel database, the thickened-skin pixel database and the KNN classifier are now applied to the pixels in the second HS image. None of the 14 tumors in this image (Fig. 2) were used in training. We do not expect our feature selection algorithm to detect tumors 4 and 5, because their lesion regions contain only eight pixels. Fig. 10 shows the binary classification image after post processing obtained for HS image 2 using the lesion features. Tumor number 4 (left center) in Fig. 2 was missed as expected; it lies on the side of the chicken and has a small lesion region (only 8 pixels), and only four of these pixels were detected by our lesion feature selection algorithm; and it was later removed by out post-processing rule (thus, it is not present in Fig. 10). Tumor number 5 in Fig. 2 is also missed. It is small and also lies on the side of the chicken carcass; our feature selection algorithm detected only 2 of its 8 pixels. We expect to miss these tumors, since we omit any pixel blob with 12 or less pixels in order to reduce false alarms. In Fig. 10, we detected 11 of the 14 tumors using lesion features; these are marked by rectangles; but 17 false alarms are also present. Thus, use of the thickened-skin pixel features is again necessary to reduce false alarms. Fig. 11 shows the binary classification image using the thickened-skin features after post processing. It has more false alarms than the image for the lesion features (Fig. 10). Only 2 thickened-skin pixels were detected on tumors 4 and 5 (in Fig. 2) and they are thus removed in our blob analysis leading to Fig. 11. In Fig. 11, we detected 12 of the 14 tumors; they are marked by rectangles. The 2 missed tumors (numbers 4 and 5 on the left side) are expected, as discussed earlier. Fig. 12 shows the final fused classification image result for this second HS image after fusion of Figs. 10 and 11. As seen, 
11 of the 14 skin tumors are detected and marked by rectangles with no false alarms. Fusion of the binary classification results reduces the number of false alarms from 17 in Fig. 10 and 60 in Fig. 11 to zero. Tumor 13 (lower right) is detected by the thickened-skin features (Fig. 11) but is missed by the lesion features (Fig. 10). The tumor has a small lesion region (16 pixels), but only 10 of these pixels are detected by our lesion feature selection algorithm and it was thus removed by our blob analysis in Fig. 10. As a result, it is not detected in the final fused classification image in Fig. 12. Tumors (4 and 5) were missed, since they have small lesion regions (as noted earlier).

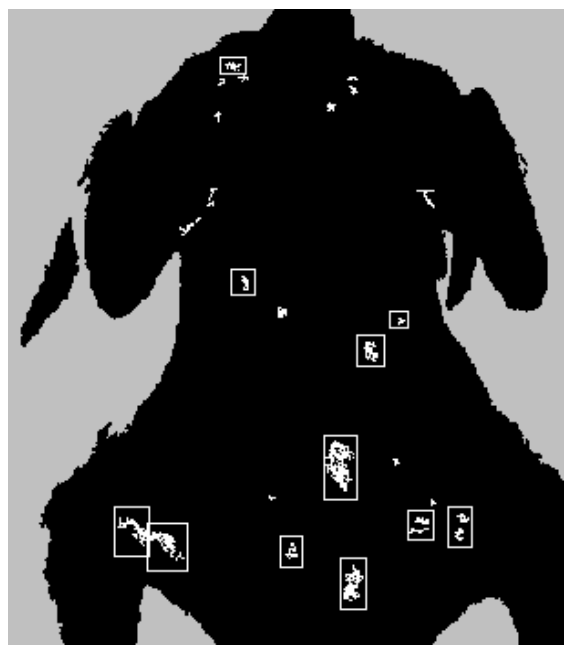

Figure 10: Detection result for the lesion features on the second HS image with detected tumors marked by rectangles.

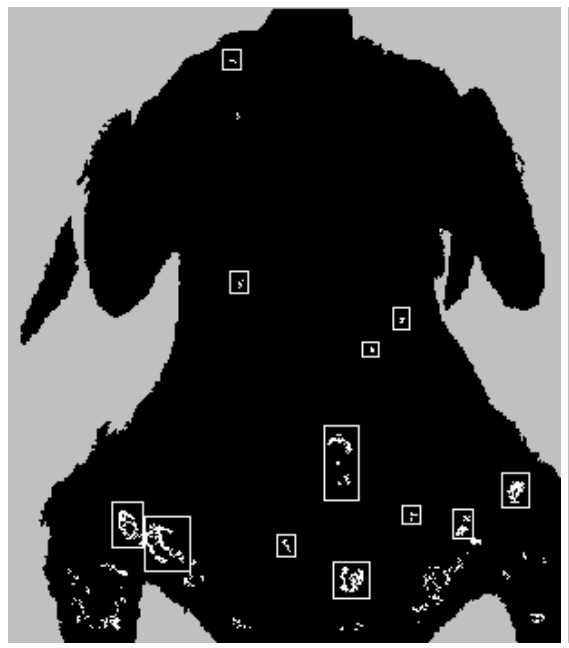

Figure 11: Detection result for the thickened-skin features on the second HS image with detected tumors marked by rectangles.

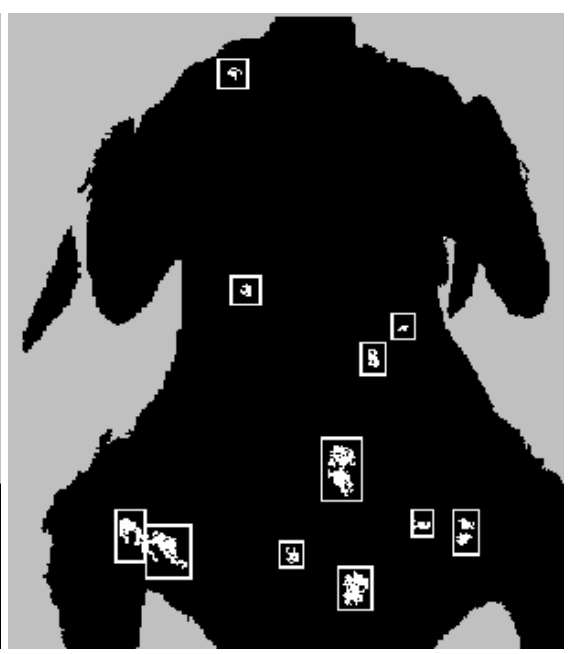

Figure 12: Final fused classification image result on the second HS image with detected tumors marked by rectangles.

\section{CONCLUSIONS}

Since the spectral responses on the lesion and thickened-skin portions of tumors are different, we train our feature selection algorithm to detect lesion and thickened-skin regions separately; we then process the resultant images, and we fuse the two detection results to reduce false alarms. Our recent FS/MBB feature selection algorithm was used. HS data was shown to be useful for detecting chicken skin tumors. Our feature selection algorithm used only 5 bands (features 11, 16, 18, 29, and 33) of HS data, thus making a fast and inexpensive HS sensor system possible. Our initial test result is promising with 18 of 21 skin tumors detected with only 2 false alarms. Others have performed much worse on such chicken images. Two of the three misclassified tumors are very small or have small lesion and thickened-skin regions and thus were expected to be missed (for data at the present resolution). One of the three misclassified tumors was detected in the thickened-skin features but missed in the lesion features .

Much more extensive tests are needed on much more data. The database should also consider higher resolution, so that there are more pixels on each tumor. Creating a training and test set database is difficult because exact pixel tumor locations and sizes are not clear in the present data. Their locations should be carefully addressed.

\section{ACKNOWLEDGEMENT}

The authors would like to thank Dr. Yud-Ren Chen, Dr. Moon Kim and Dr. Kevin Chao of the Agricultural Research Service in Maryland for providing the database used in this paper and for helpful technical discussions.

\section{REFERENCES}

[1] B. Thai, and G. Healey, "Invariant subpixel target identification in hyperspectral imagery," Proc. SPIE, vol. 3717, pp. 14-24, 1999.

[2] T. Nichols, J. Thomas, W. Kober, and V. Velten, "Interference-invariant target detection in hyperspectral images," Proc. SPIE, vol. 3372, pp. 176-87, 1998.

[3] J. Goutsias, and A. Banerji, "A morphological approach to automatic mine detection problems," IEEE Trans. Aerospace and Electronic Systems, vol. 34, No. 4, pp.1085-1096, 1998

[4] M.J. Muasher, and D.A. Landgrebe, "The K-L expansion as an effective feature ordering techniques for limited training sample size,” IEEE Trans. Geosci. Remote Sensing, vol. GE-21, pp. 438-441, Oct. 1983. 
[5] D. Casasent, and X.-W. Chen, "Waveband selection for hyperspectral data: optimal feature selection,” Proc. SPIE, vol. 5106, April 2003.

[6] T.C. Pearson, D.T. Wicklow, E.B. Maghirang, F. Xie, and F.E. Dowell, "Detecting aflatoxin in single corn kernels by transmittance and reflectance spectroscopy,” Trans. of the ASAE, vol.44(5), pp.1247-1254, 2001.

[7] D. Casasent, X.-W. Chen, and S. Nakariyakul, "Hyperspectral methods to detect aflatoxin in whole kernel corn," Mycopathologia, vol. 157(4), pp. 422, May 2004.

[8] F. Dowell, T. Pearson, E. Maghirang, F. Xie, and D. Wicklow, "Reflectance and transmittance spectroscopy applied to detecting fumonisin in single corn kernels infected with Fusarium verticillioides," Cereal Chem. vol. 79(2), pp. 222-226, 2002.

[9] F. Dowell, M. Ram, and L. Seitz, "Predicting scab, vomitoxin, and ergosterol in single wheat kernels using nearinfrared spectroscopy,” Cereal Chem., vol. 76(4), pp.573-576, 1999.

[10] Y.-R. Chen, W. Hruschka, and H. Early, "On-line inspection of poultry carcasses using visible/near-infrared spectrophotometer,” Proc. SPIE, vol. 3544, pp. 147-155, 1998.

[11] J. Heitschmidt, M. Lanoue, C. Mao, and G. May, "Hyperspectral analysis of fecal contamination: a case study of poultry,” Proc. SPIE, vol. 3544, pp. 134-137, 1998.

[12] W.R. Windham, B. Park, K.C. Lawrence, and D.P. Smith, "Analysis of reflectance spectra from hyperspectral images of poultry carcasses for fecal and ingesta detection,” Proc. SPIE, vol. 4816, pp. 317-324, 2002.

[13] A. Jain, R. Ruin, and J. Mao, "Statistical pattern recognition: a review," IEEE Trans. Pattern Analysis and Machine Intelligence, vol. 22(1), pp. 4-37, 2000.

[14] B.W. Calnek, H. John Barnes, C.W. Beard, W.M. Reid, and H.W. Yoder, Diseases of poultry, Chapter 16. pp. 386484, Iowa State University Press, Ames, IA.

[15] K. Chao, P.M. Mehl, and Y.R. Chen, "Use of hyper- and multi-spectral imaging for detection of chicken skin tumors," Applied Engineering in Agriculture, vol. 18(1), pp. 113-119, 2002.

[16] I. Kim, Y.-R. Chen, M. Kim, and S. Kang, "Application of hyperspectral fluorescence imaging for detection of skin tumors on chicken carcasses,” in 2002 ASAE Annual International Meeting, Paper No. 023142, 2002.

[17] P. Narendra, and K. Fukunaga, "A branch and bound algorithm for feature subset selection," IEEE Trans. Comput., vol. 26, pp. 917-922, 1977.

[18] K. Fukunaga, Introduction to Statistical Pattern Recognition, Academic Press Inc. New York, $2^{\text {nd }}$ Ed., 1992.

[19] M.S. Kim, Y.R. Chen, and P.M. Mehl, “ Hyperspectral reflectance and fluorescence imaging system for food quality and safety,” Trans. of the ASAE, vol.44(3), pp. 721-729, 2001.

[20] R. Duda, P. Hart, and D. Stork, Pattern Classification, $2^{\text {nd }}$ ed., A Wiley-Interscience Publication, New York, p. 48, 2001.

[21] T. Cover, and J. Campenhout, “On the possible orderings in the measurement selection problem," IEEE Trans. Systems, Man, and Cybernetics, SMC-7(9), pp. 657-661, 1977.

[22] D. Ballard, and C. Brown, Computer Vision, Prentice-Hall, Englewood Cliffs, N.J., p. 151, 1982.

[23] D. Casasent, and X.-W. Chen, "Feature reduction and morphological processing for hyperspectral image data," Applied Optics, vol. 43(2), pp. 227-236, Jan 2004.

[24] T. Pearson, "Spectral properties and effect of drying temperature on almonds with conceal damage," Lebensm.Wiss. U.-Technol, vol. 32, pp. 67-72, 1999. 